\title{
The curriculum
}

\author{
Pier Giuseppe Rossi ${ }^{1}$
}

\begin{abstract}
The complexity of socio cultural contexts and, consequently, of the school requires an explicit planning that can offer the educational action a rhizomatic and generative vision. The last-century models of the curriculum were characterized by a linear logic; however, in recent years, they have lost much of their effectiveness and cannot have any longer a positive impact over the complexity of the schooling process.

Reflecting on the curriculum means catching the characterizing elements of the current contexts at the educational level and reflecting on the elements of continuity and discontinuity from the past that could facilitate the change from linear models to recursive models.

The present article is organized into two parts: in the first one there is a comparison between the present and the past, while in the second one there is a review of the main contributions that from the Seventies of the last century have provided the basis to define the role and the structure of the curriculum. The article "the curriculum, the macro design and the micro design, and the curriculum transposition, present in the same issue, is the logic continuation of the present contribution.
\end{abstract}

Keywords: curriculum, design, complexity.

\section{Dal programma al curricolo}

Il dibattito sul curricolo ha avuto un periodo felice e ricco nella seconda metà dello scorso secolo. Il dibattito partiva dalla necessità di organizzare una cornice di senso al percorso educativo coniugando le finalità educative con gli obiettivi didattici. Occorreva superare l'ottica dei programmi la cui struttura era la successione dei contenuti disciplinari per approdare a una visione coerente

${ }^{1}$ Pier Giuseppe Rossi is Full Professor on Didactics and Special Pedagogy, Department of Education, Cultural Heritage and Tourism, University of Macerata. 
con la ricerca didattica di quel periodo che vedeva nell'instructional design e in una didattica per obiettivi i riferimenti principali. Nei programmi della scuola elementare del 1985 (D.P.R. 12 febbraio 1985, n 104) si afferma "Per attuare i suoi compiti la scuola elementare si organizza in modo funzionale rispetto agli obiettivi educativi da perseguire". Sempre in tale documento si afferma:

La programmazione didattica ha un valore determinante per il processo innovativo che, con i programmi, si deve realizzare nella scuola elementare. Spetta ai docenti, collegialmente ed individualmente, di effettuare con ragionevoli previsioni la programmazione didattica, stabilendo le modalità concrete per mezzo delle quali conseguire le mete fissate dal programma e la scansione più opportuna di esse, tenuto conto dell'ampliamento delle opportunità formative offerte dal curricolo, sia con l'inserimento di nuove attività, sia con la valorizzazione degli insegnamenti tradizionali. La programmazione, nel quadro della prescrittività delle mete indicate dal programma, delineerà i percorsi e le procedure più idonee per lo svolgimento dell'insegnamento, tenendo comunque conto che i risultati debbono essere equivalenti qualunque sia l'itinerario metodologico scelto. La programmazione didattica deve essere assunta e realizzata dagli insegnanti anche come sintesi progettuale e valutativa del proprio operato.

Dal programma, che indicava solo il cosa, si passa al curricolo che indica anche il come, anche se ancora in rapporto biunivoco e strumentale al cosa o all'obiettivo. I Programmi sottolineano la necessità della programmazione, dall'altro esplicitano come essa permetta di realizzare gli obiettivi previsti per ciascuna disciplina. Il curricolo si struttura principalmente in macro-obiettivi che servono da guida per l'articolazione della micro-progettazione. Poiché l'obiettivo si presta a un processo di suddivisione riduzionista, ovvero dato che vi è la possibilità di articolare il macro-obiettivo in una serie di micro-obiettivi, il passaggio dalla macro alla micro-progettazione può essere descritto come un processo di suddivisioni successive. A partire dai macro-obiettivi, presenti nel curricolo, si procede alla individuazione dei micro-obiettivi e alla articolazione della micro-progettazione.

Anche se si parla di programmazione, la legge 104 segna una rottura con i precedenti approcci basati sui contenuti e, soprattutto nelle premesse, esplicita finalità sociali e civili, e l'attenzione allo studente e a processi di personalizzazione e inclusione.

Simile risulta, comunque, la logica che lega macro e micro-progettazione. Le strutture sia della macro, sia della micro-progettazione sono speculari e contengono "oggetti" qualitativamente simili anche se di granularità diversa. In entrambi casi, infatti, la micro-progettazione è un frammento di un contenuto/ obiettivo macro. La relazione tra programma/curricolo e sessione/lezione è lineare. Il livello macro fornisce indicazioni precise su cosa trattare nei moduli 
e nelle sessioni di lavoro e, soprattutto, i mattoni che costituiscono la macro e la micro progettazione sono dello stesso tipo anche se di granularità diversa. Nei programmi per contenuti i mattoni della macro e della micro-progettazione sono argomenti e temi della disciplina: in fisica l'argomento macro "studio del moto" si articola in temi quali "velocità" o "moto rettilineo uniforme", in letteratura italiana l'argomento "Romanticismo" si declina in "Leopardi" o nello studio di un singolo idillio. Ugualmente nei programmi per obbiettivi il macro-obiettivo "saper produrre un testo minimo" si articola in microobiettivi quali "saper produrre un testo argomentativo" o "conoscere la regola grammaticale". Tra macro e micro varia la granularità, ma non la tipologia.

La similitudine tra programmi per contenuti e curricoli per obiettivi, ovvero la presenza di una logica prioritaria e prevalente determina una relazione lineare tra macro e micro, e deriva da una minore complessità del sapere e del contesto socio-culturale. Inoltre tra obiettivi e contenuto disciplinari vi era un legame biunivoco. Gli obiettivi possono essere visti come il prodotto della trasposizione didattica, più che un oggetto cognitivo autonomo dai contenuti.

\section{La logica del non-uno}

Il panorama attuale è completamente differente. La logica del Non-uno (Braidotti, 2013) prende forma anche in campo educativo dove l'agire didattico si articola intorno a differenti elementi tra loro non riducibili a logiche uniche o univoche. Il sapere disciplinare rimane una presenza fondante dell'agire didattico. Emerge, poi, il costrutto di competenza, sicuramente proteiforme. Può essere ridotto a traguardo, ma in tal modo si fornisce un'immagine limitata, sfuocata e cristallizzata. Può essere visto come orchestrazione in contesto di conoscenze, procedure e abilità. In tal modo si evidenzia la connessione con i processi e la situazione e, quindi, la possibilità di reificarsi con forme e modalità differenti in differenti contesti e soggetti. Può essere visto, infine, come un sapere-strumento, come un filtro con cui analizzare il fare dei soggetti e cogliere la consapevolezza con cui essi analizzano le proprie pratiche e le modalità personalizzate e non prevedibili con cui risolvono situazioni problematiche.

Oltre ai contenuti disciplinari e alle competenze, vi è un terzo elemento che caratterizza i contesti scolastici, differenziando la situazione attuale dalla passata. Gli studenti arrivano in classe con un ricco bagaglio non formale in cui sono presenti non solo, come ieri, esperienze di vita, ma anche contenuti appresi attraverso il linguaggio e l'uso di artefatti digitali. Sono saperi che hanno strutture differenti da quelle del sapere sapiente in quanto spesso sono a macchia di leopardo e non sono validati, ovvero non sistematici. Nello stesso 
tempo, tali saperi sono differenti da quelli che Vygotskij (1954) chiama saperi quotidiani in quanto non sempre derivano dell'esperienza, ma da processi di mediazione e hanno elementi tipici del sapere sapiente, quali l'utilizzo del linguaggio simbolico scritto.

Gli effetti della presenza di artefatti digitali nelle pratiche, soprattutto non formali, degli studenti e nelle pratiche professionali va analizzata in profondità. Nel mondo analogico la relazione tra soggetto e mondo era supportata da artefatti che nell'uso divenivano trasparenti, quali il martello del ciabattino, la falce del mietitore o lo stetoscopio del medico, rendendo la teoria un processo di riflessione, strutturazione e generalizzazione, spesso diacronico con la pratica stessa. Si studiava prima dell'azione e si rifletteva, poi, sistematizzando le strutture teoriche. Oggi il digitale pone tra soggetto e mondo schermi e artefatti popolati da simboli il cui valore è determinato da input provenienti da sensori e dalle regolazioni operate del soggetto. Sono spesso rappresentazioni digitali che forniscono indicazioni relative al mondo circostante o valori selezionati dal soggetto per scegliere specifiche procedure. $\mathrm{Se}$ in un forno elettrico vi è ancora una relazione analogica fra l'angolo di rotazione della manopola, che seleziona la temperatura, e la temperatura stessa, nelle moderne macchine per la panificazione domestica i programmi 1 o 2 o 3 corrispondono a una successione di stati ciascuno caratterizzato da una durata, da una temperatura e da un moto della paletta. La corrispondenza tra numero e procedura è convenzionale e comprensibile solo leggendo le istruzioni dei manuali.

Hoadley e Jansen (2011, 190), partendo dalle teorie di Vygotskij e Bernstein, ritengono essenziale per l'educazione nel mondo attuale un maggior rapporto tra conoscenza esperienziale e conoscenza scolastica e individuano, tra le altre, alcune caratteristiche proprie della conoscenza che nasce dall'esperienza quotidiana (everyday life): è (1) orale, (2) basata su opinioni locali e legate al contesto e (3) connessa alla pratica e al concreto, ovvero è ancorata a un particolare contesto. Può la conoscenza extrascolastica oggi essere connessa in modo esclusivo a queste tre caratteristiche? Essa è sempre più mediata da simboli scritti ed è frutto di esperienze non dirette e, spesso, decontestualizzate.

La presenza di media digitali, nelle prassi quotidiane e negli ambienti di vita, produce una moltiplicazione di saperi e la scuola, in termini quantitativi, non è più l'unica o la principale dispensatrice di un sapere veicolato da un linguaggio simbolico. Tutto ciò modifica l'insegnamento che da processo che dal sapere sapiente costruisce il sapere insegnato, sta diventando il processo che supporta lo studente nell'aggregazione (Kress, 2015) dei frammenti sia appresi in contesti non-formali, sia in contesti formali per arrivare alla costruzione di un senso proprio. Come afferma Duit (2007): 
[In the Didaktik tradition] both processes "elementarization" and "construction of the content structure for instruction" are intimately interrelated to decisions on the aims of teaching the content and the students' cognitive and affective perspectives.

These perspectives include students' pre-instructional conceptions and their general cognitive abilities on the one hand and their interests, self-concepts and their attitudes on the other (Duit, 2007, 6).

La logica del Non-uno, oltre che dai saperi, deriva anche dalle altre diversità presenti nella scuola: culture, abilità, conoscenze, che non possono essere inserite in una scala unica e con precise gerarchie. Esse possono essere fonte, a un tempo, di difficoltà o di ricchezza e ciò dipende dai contesti e dalle interazioni che si attivano tra le stesse. Inoltre la presenza di tali differenze va vista non solo come situazione di partenza, poiché le pratiche in classe favoriscono a un tempo la presenza di elementi di tangenza e di condivisione, ed elementi di personalizzazione del sapere stesso (Massa, 2004).

Fig. 1 - Dal lineare al ricorsivo

Macro-contenuto/macro-biettivo

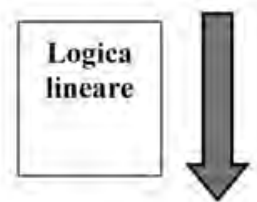

Macro-contenuto/macro-obiettivo
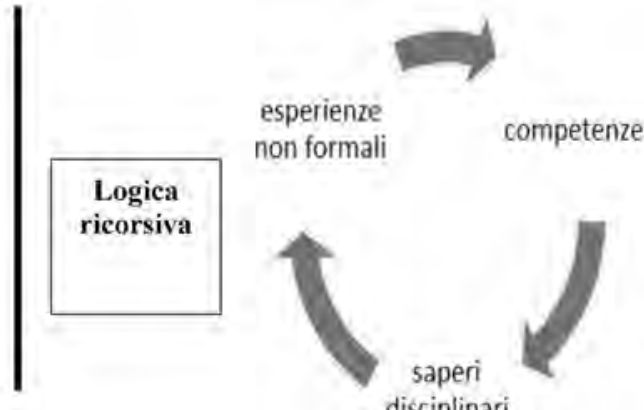

disciplinari

\section{I riferimenti teorici}

Il dibattito sul curricolo è ampio e molto ricco. In questa sede si analizzano solo alcuni passaggi significativi.

\section{Stenhouse}

Il volume di Stenhouse "Dalla scuola del programma, alla scuola del curricolo" (1991) è la sintesi del ricco dibattito sviluppatosi sul tema negli anni '60 e '70. Riporta alcune delle definizioni di curricolo della letteratura 
di allora. Dalla definizione di Johnson (1967): "si conviene qui intendere il curricolo come una serie articolata d'esiti culturali prestabiliti. Il curricolo fissa in anticipo (o almeno si ripromette) i risultati dell'apprendimento" (ivi, 130) si passa a quelle di Inlow (1966): "il curricolo è il programma composito elaborato da ciascuna scuola al fine di orientare verso esiti prestabiliti l'apprendimento degli allievi" (ivi, 7), di Neagley ed Evans (1967) "il curriculum è la programmazione d'un complesso d'esperienze elaborate dalla scuola affinché gli alunni conseguano gli esiti culturali previsti al massimo delle loro capacità" (ivi, 2). Stenhouse propone poi la seguente definizione:

Un curriculum è un tentativo di comunicare i principi e le caratteristiche essenziali d'una proposta educativa in forma tale da restare aperto a qualsivoglia revisione critica e suscettibile di un'efficiente conversione in pratica (Stenhouse, 1991, 18).

Dall'analisi delle pratiche e della letteratura individua le due tipologie di curricolo:

da una parte esso si configura come intenzione, programma o indicazione, cioè come idea di ciò che si auspica possa ottenersi a scuola, dall'altra rappresenta invece un quadro della reale situazione scolastica, di ciò che in effetti avviene (ivi, 16).

Le due tipologie possono, come si illustrerà successivamente, convivere. Ad esempio si potrà avere, come nella situazione italiana, il Curricolo di istituto che si configura come idea in base a cui costruire i percorsi didattici e le progettazioni annuali di classe dei docenti che ipotizzano la struttura del percorso annuale.

Stenhouse suggerisce, poi, alcuni principi per strutturare un curricolo, principi validi ancora oggi:

Come programma minimo, un curricolo dovrebbe fornire la base per pianificare un corso, studiandolo da un punto di vista empirico e considerandone i fondamenti che lo giustificano. Esso dovrebbe offrire:

a) Nella pianificazione:

1. i principi per la scelta dei contenuti: cosa si deve insegnare e imparare;

2. principi per lo sviluppa d'una strategia didattica: come si deve insegnare a imparare;

3. principi con cui diagnosticare validità o carenze dei singoli studenti e differenziare i principi 1, 2, 3 per far fronte a casi particolari.

b) Nello studio empirico:

1. principi in base a cui studiare e valutare il progresso degli studenti;

2. principi in base a cui studiare e valutare il progresso degli insegnanti;

3. consigli circa le modalità d'adottare il curriculum presso contesti variabili e di scuola e d'allievi, situazioni ambientali e gruppi d'individui coetanei; 
4. informazioni circa la varietà degli effetti presso contesti diversi e su allievi differenti, e spiegazioni circa le cause di tale varietà.

c) Riguardo alla giustificazione:

1. una formulazione degli intendimenti o obiettivi del curriculum che sia suscettibile di revisione critica (ivi, 19).

Va precisato che oggi l'analisi dei due primi principi non sarebbe fatta separatamente, ma si esaminerebbe come essi interagiscono all'interno di un dispositivo o di un'attività.

L'analisi di Stenhouse si focalizza poi sul modello, allora più diffuso, di curricolo, quello per obiettivi, e ne evidenzia punti di forza e di debolezza. L'analisi risulta quanto mai attuale. Il punto di forza è l'utilità di individuare e descrivere in modo accurato i comportamenti per un insegnamento efficace e la facilità di una valutazione oggettiva. Ma proprio da tale "facilità" derivano i due limiti: ci si concentra su obiettivi praticabili ovvero facilmente valutabili mettendo in secondo piano alcune importanti finalità della scuola, come ad esempio la padronanza di alcuni processi. Da qui discende, in primis, l'analisi di una proposta alternativa ovvero il modello di programmazione e sviluppo del curricolo centrato sui processi, soprattutto su basi logiche. Stenhouse non elimina il ricorso a percorsi per obiettivi, ma cerca di cogliere i contesti educativi in cui attuarlo. Egli sostiene che un curricolo centrato sui processi

è più adatto del modello per obiettivi in quelle parti del curriculum che s'incentrano sulla conoscenza e sulla comprensione. Il modello degli obiettivi sembra convenire meglio ai settori curricolari che danno più risalto alle informazioni e alle attitudini pratiche (ivi, 124).

Si concentra, poi, su una seconda critica al curricolo per obiettivi: la sottovalutazione della professionalità dei docenti. Questo, forse, è il punto focale di tutto il testo. La didattica per obiettivi, come per altro i modelli prescrittivi di Instructional design quali ADDIE, sembrano ipotizzare la possibilità di processi che funzionano indipendentemente dalla qualità del docente. Anche i processi valutativi della didattica per obiettivi, basati su criteri più che su norme, sembrano sortire lo stesso effetto. A riprova della analisi precedente, Stenhouse sottolinea che in una programmazione antitetica a quella per obiettivi, quella che adotta il modello processuale "il punto debole dovrebbe essere evidente. Tutto dipende dalla qualità dell'insegnante; ma questa ne è anche la forza più grande" (ivi, 123). E, più avanti: "Il modello per obiettivi, applicato ai campi della conoscenza, mi sembra che punti soprattutto a migliorare l'insegnamento come istruzione senza però migliorare la sapienza o preparazione del docente" (idem). Purtroppo o per fortuna la centralità del ruolo dell'insegnante nella didattica è un punto fermo dell'insegnamento 
formale, sia face to face, sia a distanza. È attualmente evidenziata anche da ricerche e proposte didattiche, quali quelle di Damiano (2013) e di Hattie (2008). Pertanto la ricerca della qualità non può che basarsi sulla professionalizzazione dei docenti, soprattutto in un contesto di complessità dove si richiede ai docenti di progettare i percorsi in base ai contesti. Non si crede possa sortire effetti positivi pensare alla qualità con soluzioni che meccanicizzano il processo.

Stenhouse, nell'ottica di un docente professionista, propone la strada del insegnante-ricercatore. Per un docente il compito principale non è quello di applicare delle procedure, ma apprendere dalla propria pratica e inserirsi in un processo di LifeLong Learning. Il ricercatore inglese afferma che gli insegnanti "sanno quello che fanno", elemento questo sottovalutato dai cultori di una didattica per obbiettivi e non sottovaluta che la proposta di in insegnante-ricercatore deve tener conto delle reali conoscenze e competenze possedute. I docenti non possono avere su ogni tema un sapere esperto. Da tale considerazione deriva che l'insegnante potrà essere "o un esperto o anch'egli uno che apprende. In molti casi particolari, l'insegnante non può essere un esperto e, per conseguenza, deve ripiegare sul ruolo di apprendista" (ivi, 117), ma questo non contrasta con una postura di ricerca.

\section{Bernstein}

Gli studi sul curricolo di Bernstein si distribuiscono su periodo molto ampio che inizia dagli anni '50 del secolo scorso fino ad arrivare alla prima decade del presente millennio.

L'analisi di Bernstein, che si colloca in una prospettiva strutturalista, evidenzia come la costruzione del curricolo ricalchi una prospettiva di controllo e di potere e associa a ciò i processi di classification e framing (1981) che caratterizzano la struttura di ogni curricolo.

Classification refers to the degree of maintenance of boundaries between categories (subjects, spaces, discourses). Classification is strong when there is a sharp separation between categories, this originating hierarchies in which each category has a specific status and voice and therefore a given power; classification is weak when there is a blurring of boundaries between categories.

Framing refers to the social relations between categories that is to communication between these categories. Framing is strong when the categories with higher status have the control in that relation and is weak when the categories with lower status also have some control in the relation. Between the extremes of strong and weak classifications and framings, a grading may exist.

However, behind a given classification and framing are, respectively, the power relations and the fundamental principles of social control. Power maintains the classification that is the insulations, the boundaries between "things", be them internal or external to the subject. 
The modality of socialisation, that is the pedagogic interaction, is regulated by the strength of framing. However, because power can be realised through framings of distinct strengths, a situation may occur in which power relations are not altered but they are realised through a change in the form of socialisation (Morais e Neves, 2007).

Per Bernestein classification e framing forniscono una chiave interpretativa con cui analizzare le strutture del curricolo. Non vi è una meccanica connessione fra i livelli di classification e framing e la qualità della didattica, poiché spesso solo in connessione con il contesto è possibile vedere come $\mathrm{i}$ due meta-concetti operano nella didattica. Spesso una classification e framing "strong" favoriscono processi educativi volti al mantenimento di una struttura culturale e sociale, in quanto Bernstein ritiene che la "insulations" (strong classification) e una rigida gerarchia (strong framing) non favoriscano una flessibilità e mobilità dei saperi e una mobilità e democratizzazione sociale.

From a theoretical point of view, this means that in a society characterised by an equitative distribution of power and by principles of control based on horizontal/open social relations, the dominant code is a code which legitimates weak classification and framings (idem).

Ma, all'opposto, una struttura leggera sottende elementi impliciti e una tale situazione favorisce chi già conosce le regole del gioco educativo, ovvero chi per famiglia di appartenenza è educato allo studying ${ }^{2}$.

L'attenzione alle frontiere tra le discipline e alle relazioni tra le stesse, su cui si focalizza Bernstein, è di fondamentale importanza mentre si progetta il curricolo. In una didattica per competenze, focalizzata sulle attività, l'attenzione alla interrelazione tra i saperi sembrerebbe garantita in quanto le attività e le pratiche, per costrutto ontologico, presentano situazioni problematiche che richiedono per la loro soluzione l'interazione tra più saperi disciplinari. Nello stesso tempo richiedono una maggiore autonomia dello studente, autonomia per la quale lo studente deve essere formato.

Classification e framing vanno a impattare sulle modalità più o meno meccaniche, più o meno riflessive con cui le teorie e le procedure richiamate dialogano con i contesti e le soluzioni. Un processo meccanico e non riflessivo dei saperi disciplinari equivale a una rigidità e isolamento dei saperi stessi e nasce da un riconoscimento aprioristico e non storicizzato degli stessi. Un approccio leggero equivale invece a processi ricorsivi in cui le pratiche producono riflessioni generative e circolari sulle teorie e viceversa, e le stesse

\footnotetext{
${ }^{2}$ Studiyng è un termine inglese non traducibile in italiano che descrive la postura necessaria per lo studio, la conoscenza di comportamenti e di regole spesso implicite connesse con la vita scolastica e lo studio.
} 
hanno validità non in sé e per sé, ma nella capacità di interagire con i contesti, di essere generative, ovvero di essere a un tempo strutturanti dei contesti, ma anche da essi strutturate.

Già Bernstein aveva colto che:

The codes integrate two aspects and give the subjects the possibility of making their reading and creating the texts which can be legitimately constructed, or texts which are within the possibilities of the syntaxes of generation and realisation, as ortodox/ heterodox potential texts (idem).

La ricerca di Bernstein si sviluppa nel decennio successivo sulle tematiche di "generation and acquisition/transformation of codes" (1986) e cerca di rendere esplicite le relazioni tra livelli istituzionali macro e micro.

In this way, the transmitters and the acquirers constitute specialised categories with specialised voices. From the point of view of the acquisition of the voice, the distinction of categories provides a set of criteria of demarcation that permit the recognition of categories in the variability of its presentation and give to the subject the basis to infer the recognition rules.

These rules regulate what can be associated, that is which meanings can be legitimately associated, therefore regulating the principles for the generation of legitimate meanings, and in this way they create what can be designated by syntax of generation of the legitimate meanings. In this way, a relation can be established between the distribution of power (external to the subject) and the syntax of the generation of meanings (internal to the subject) (idem).

La relazione tra acquisizione e trasformazione è legata, da un punto di vista epistemologico, alla struttura disciplinare e alla "ortodossia", da un punto di vista pedagogico-didattico allo spazio di autonomia e di attività del soggetto.

\section{Joannert}

Joannert e Van der Borght (2009) si muovono in una prospettiva sociologico - politica e hanno come sfondo la comparazione tra modelli anglofoni, dove il curriculum mantiene il significato originario (Bobbit, 1918) ovvero quello di piano d'azione pedagogico, e modelli francofoni, dove invece il curricolo è identificato con i programmi scolastici, tratta i saperi, le discipline, i contenuti e la loro strutturazione. Se pertanto il modello anglofono si pone a un livello meta, quello franco-europeo è si intreccia operativamente e nei contenuti con il percorso didattico e il programma di studi.

Per gli autori il curricolo è un dispositivo stratificato a più livelli (Keeves, 1992), non lineare ma sistemico (Fig. 1). Tale configurazione dovrebbe garantire dinamismo, inteso come ricorsività tra livelli, anche se l'autore 
lascia la questione aperta, si chiede se tale movimento tra livelli sia realmente possibile.

Joannert individua tre livelli: il livello del curricolo prescritto (intended curriculum), il livello del curricolo e dei programmi insegnati (achieved curriculum), il livello degli apprendimenti scolastici e dei loro risultati (implemented curriculum), da cui emerge una specularità con il processo di trasposizione didattica di Chevallard (1985) e, forse più, con il modello di Develay.

In base a tale ipotesi il sapere viene codificato nel "cammino curricolare" e si potrebbe parlare di "trasposizione curricolare" (Joannert e Van der Borght, 2009), entro cui il sapere "va e viene incessantemente tra i tre livelli" (p. 139).

Questa ricorsività tra $\mathrm{i}$ tre livelli e la necessità di un curricolo trasposto mette in luce la complessità del curricolo che diviene un artefatto continuamente in divenire, grazie alla mediazione continua dell'insegnante.

Vi è una terza domanda a cui l'autore cerca di rispondere: è possibile analizzare razionalmente uno strumento di per sé irrazionale perché pensato e situato nelle dinamiche irrazionali della società di riferimento? Ovvero come si può analizzare un tale curricolo? È sufficiente suddividerlo in strati e costruire appositi strumenti? Può tale analisi restituirne la complessità e il dinamismo?

L'autore fa una disamina di varie modalità di analisi del curricolo che tuttavia, a suo avviso, hanno il limite di focalizzarsi su uno solo dei livelli, in genere quello del prescritto, ma non riescono a cogliere il curricolo realizzato in classe e tanto meno la ricorsività tra $\mathrm{i}$ tre livelli.

Ciò deriva - o almeno questa è l'ipotesi dell'autore - dall'assenza di una relazione effettiva tra un livello e l'altro, intesa come movimento di andata e ritorno in cui i tre livelli si influenzino reciprocamente. Secondo l'autore la difficoltà deriva da una mancata centratura del curricolo sull'apprendente, inteso come membro di una società dalle dimensioni multiple. Da qui deriva l'attenzione, soprattutto in una realtà complessa, sul percorso di trasposizione curricolare più che sul curricolo in quanto tale, sul processo nella sua ricorsività, più che sull'artefatto. Introduce il concetto di curricolo trasposto.

Tale analisi riporta l'attenzione su alcuni elementi precedentemente evidenziati: da un lato l'esigenza di una progettazione articolata ed esplicita, mentre, precedentemente, la presenza di una dimensione prevalente nella struttura di un curricolo permetteva approcci taciti e impliciti, e, connesso a ciò, l'esigenza di una trasposizione e di una co-progettazione, come si argomenterà nei successivi paragrafi.

\section{Hoadley e Jansen}

La ricerca pluriennale di Hoadley (2012), pur muovendosi in un'ottica fortemente connessa al contesto in cui opera, offre spunti e indicazioni che possono essere generalizzati. Il punto di partenza è la constatazione che: 
We have also found that there is a gap between the curriculum plan and the practices of teachers. This is sometimes problematic, particularly when it is a consequence of teaching inadequacies or rigidities. But at other times, the gap is, in fact, the space in which a teacher innovates and improves a curriculum plan (idem).

L'origine di tale gap nasce dal fatto che "the curriculum, like schooling, is intensely political" e "the choices of the plan's content and form are often made on the basis of the beliefs of certain groups - the state, unions or labour, or academics", mentre "teachers' beliefs determine how they teach, and which bits of content they emphasize, and, ultimately, the choices made in terms of plan and practices have consequences for learners".

Nella prospettiva degli autori il curricolo viene visto come organizzazione della conoscenza e questo incide sia sulla scelta degli argomenti, sulla organizzazione degli stessi e sulla loro interazione ("how different subjects, and different topics within a subject fit together") da cui emerge il forte riferimento a Bernstein (1996). Sempre partendo dai modelli del sociologo inglese, gli autori descrivono due distinti approcci al curricolo: il curricolo per competenze e quello per performance (Tab. 1), anche se poi le pratiche dei docenti, come gli stessi autori ammettono, presentano modelli ibridi.

Tab. 1 - Confronto tra modello per competenze e modello per performance.

\begin{tabular}{|l|l|}
\hline Model & Characteristics \\
\hline Competence & $\begin{array}{l}\bullet \text { characterized by the idea of integration between subjects } \\
\bullet \text { makes strong links between school learning and real life }\end{array}$ \\
\hline Performance & $\begin{array}{l}\bullet \text { stresses the importance of separate subject disciplines } \\
\bullet \text { does not draw extensively from real life in order to teach at school }\end{array}$ \\
\hline
\end{tabular}

Gli autori evidenziano il ruolo che assume oggi nei processi educativi il rapporto tra mondo extra scolastico e attività in classe e le potenzialità di un approccio per competenze che valorizzi le esperienze già possedute dagli studenti e fornisce strumenti per operare nel mondo: "encourages teaching that draws from a learner's own experiences and 'everyday knowledge' and, in turn, assists learners in using their new learning in their lives and work".

In other words, the competence approach is learner-centred. Learners take control of their own learning, and the teacher's role tends to be covert. Rather than directly transmitting learning, the teacher acts as a guide and facilitator. Pedagogy is personalized and process-orientated. Knowledge in a competence curriculum is often horizontally organized. It introduces themes, projects and problems, which don't 
necessarily link to each other. In other words, rather than focusing overly on a curriculum that progresses vertically - where new work builds on old work, and becomes increasingly difficult - it organizes teaching around one theme, and then moves to another theme that may not be connected in any way with the first (ivi, 176).

In relazione alla valutazione l'attenzione è "on 'presences' - on what the learners know or have achieved rather than what they don't know (absences)", come invece avviene nell'approccio per performance.

L'elaborazione dei due autori va poi ad ampliare il concetto Bernsteiano di classification.

We can use the term classification to describe the relationship - or the boundary - between our 'everyday knowledge' (such as driving a car, tying your shoelaces, cooking rice) and formally taught 'school knowledge' (addition, climatology, World War II). A curriculum can be described as strongly classified if clear distinctions are drawn between what is learnt in school and what is learnt in everyday life. School systems that make very little use of everyday life experiences to teach school knowledge, or test the application of knowledge in real life, are strongly classified (ivi, 181).

Infine evidenziano due strutture possibili per il curricolo, il collection type curriculum e il integrated type curriculum:

In a collection type of curriculum there is a hierarchical (or "vertical") organization of knowledge (ivi, 183).

A collection type of curriculum is associated with the performance model of curriculum. Subject content, space, and time are strongly classified in both.

In the integrated curriculum contents of different subjects are blurred in relation to each other. This is education in breadth. There is less focus on detailed understanding of specific subjects, and much more emphasis on "horizontal" or "lateral" links across a greater range of subjects. An integrated curriculum is associated with the competence model we outlined earlier. Both are characterized by weak classification, both in terms of boundaries between subjects and boundaries between everyday knowledge and school knowledge (ivi, 184).

\section{Conclusioni}

Stenhouse ci fornisce una prima indicazione: la presenza di due tipologie di curricolo, la prima caratterizzata da regole, la seconda dalla descrizione del percorso, anche se nel suo modello è ancora forte il ruolo di un principio regolatore. Bernstein suggerisce la relazione tra curricolo e ordine socioculturale e evidenzia l'importanza del framing, ovvero della struttura 
del curricolo che è essa stessa contenuto. Joannert propone tre livelli di curricolo: il curricolo prescritto, il livello del curricolo insegnato, il livello degli apprendimenti. Inoltre introduce il concetto di curricolo trasposto. Nella reinterpretazione del pensiero di Bernstein a opera di Hoadley e Jansen viene colto il passaggio da un curricolo per performance a un curricolo per competenze e tale passaggio ci introduce al problema attuale: cosa cambia quando si passa da un curricolo che indica cosa si debba fare a un curricolo che suggerisca come decidere e scegliere cosa si debba fare, o ancora più precisamente, quando mancando un principio regolatore occorre costruire un equilibrio, una coerenza tra differenti logiche. In tale direzione può essere di aiuto Ferraris che riprendendo Searle: distingue tra le regole regolative che danno norme a una situazione esistente e quelle costitutive che la rendono possibile. Per esempio le regole degli scacchi che non mettono ordine nel traffico altrimenti caotico di alfieri, torri, pedoni, ma danno senso ad alfieri, torri, pedoni, arrocchi e scacchi-matti (Ferraris, 2011, 143).

L'analisi del contesto attuale, proposta nell'articolo, focalizza l'attenzione sulla necessità di passare a un curricolo generativo, che dia senso all'agire didattico costruendo un equilibrio e un sistema che connettano le molte prospettive e traiettorie oggi presenti. L'equilibrio e il sistema difficilmente sono riducibili a una logica unica e ordinante. L'elemento di coerenza è la rete stessa, le relazioni che si creano tra le differenti logiche. E questo è quanto si cercherà di sviluppare nell'articolo "Il Curricolo, la macro-progettazione e la micro-progettazione, e il curricolo trasposto" presente in questo stesso numero della rivista.

\section{Riferimenti bibliografici}

Bernstein B. (1996). Pedagogy,Symbolic control and Identity. London: Taylor and Francis.

Braidotti R. (2013). The Posthuman. Polity Press. Cambridge.

Chevallard Y. (1985). La transposition didactique: du savoir savant au savoir enseigné. Grenoble: La Pensé Sauvage.

Damiano E. (2013). La mediazione ddiasttica. Milano: FrancoAngeli.

Duit R. (2007). Science Education Research Internationally: Conceptions, Research Methods, Domains of Research. Eurasia Journal of Mathematics, Science \& Technology Education. 3, 1, 3-15.

Ferraris M. (2011). Anima e Ipad. Parma: Ugo Guanda.

Hattie J. (2008). Visible Learning: A Synthesis of Over 800 Meta-Analyses Relating to Achievement. NY: Routledge.

Hoadley U., Jansen J. (2012). SAIDE Curriculum. Organizing knowledge for the classroom. Oxford: Oxford University Press. 
Inlow G.M. (1966). The emergent Curriculum. New York: Wiley.

Joannert P. (2011). Curriculum, entre modèle rationnel et irrationalité des sociétés. Revue Interntionale d'éducation. 56, 135-145.

Jonnaert P., Van der Borght C. (2009). Créer des conditions d'apprentissage. Un cadre de référence socioconstructiviste pour la formation didactique des enseignants. Paris/Bruxelles: De Boeck-Université.

Johnson H.M. (1961). Sociology: a systematic introduction. London: Roudledge.

Keeves J. (1992). Methodology and measurement in international and educational surveys. London: Pergamon Press.

Kress G. (2015). Multimodalità. Bari: Progedid.

Massa R. (2004). Le tecniche e i corpi. Verso una teoria dell'educazione. Milano: Unicopli.

Morais A.M., Neves I.P. (2007). A teoria de Basil Bernstein: Alguns aspectos fundamentais. Revista Pris Educativa. 2, 2, 115-130 (ver. inglese nel sito: http:// essa.ie.ulisboa.pt/bernsteinstheory_text.htm Visitata il 16/12/2017.

Neagley R.L., Evans N.D. (1967). Handbook for Effective Curriculum Development. New York: Prentice Hall.

Rivoltella P.C. (2013). Fare didattica con gli EAS. Episodi di Apprendimento Situati. Brescia: La Scuola.

Rivoltella P.C. (2014). La previsione. Neuroscienze, apprendimento, didattica. Brescia: La Scuola.

Rossi P.G. Microprogettazione. Milano: FrancoAngeli.

Stenhouse L. (1991). Dalla scuola del programma alla scuola del curricolo. Roma: Armando.

Vygotskij L.S. (1954). Pensiero e linguaggio. Firenze: Giunti-Barbera. 